\title{
Comparison and analysis of PAPR reduction techniques in OFDM
}

\author{
Peyali Choudhury ${ }^{1}$, Achala Deshmukh ${ }^{2}$ \\ ${ }^{1,2}$ (Department of Electronics and Telecommunication, Sinhgad College of engineering/Pune university,India)
}

\begin{abstract}
In this paper, different techniques for reducing Peak To Average Power Ratio (PAPR) is discussed. The performance of an orthogonal frequency division multiplexing(OFDM) system is degraded if the peak-toaverage power ratio(PAPR) is high. An OFDM system dynamic range is typically two or four times larger than a single carrier system increasing value of the dynamic range will lead to an increased cost, power consumption of transmitter amplifier and also lead to high peak to average power ratio (PAPR). This is one of the major drawbacks of OFDM system. A serious problem of large PAPR presents when energy-inefficient nonlinear power amplifiers are used. Several techniques have been proposed to reduce PAPR in OFDM system, out of that few are selective mapping (SLM), tone reservation and partial transmit sequence. SLM method could be used to reduce PAPR, however, it causes the BER degradation and increases computational complexity. The good improvement in PAPR given by the present techniques permits the reduction of the complexity and cost of the transmitter significantly.
\end{abstract}

Keywords - Orthogonal frequency division multiplexing (OFDM), partial transmit sequence (PTS), peak to average power ratio (PAPR), selective mapping (SLM), tone reservation.

\section{Introduction}

Due to the high data rate transmission, orthogonal frequency division multiplexing (OFDM) is a promising technique in the current broadband wireless communication system. The basic principle of OFDM is to split a high-rate data stream into a number of lower rate streams that are transmitted simultaneously over a number of subcarriers. The carriers can be made orthogonal by appropriately choosing the frequency spacing between them.

The main concept of OFDM is orthogonality of the subcarriers. Since the carriers are all sine and cosine waves, we know that area under one period of these are zero. Therefore these are all orthogonal to each other. The orthogonality allows simultaneous transmission of a lot of sub carriers in a tight frequency space without interference from each other. The orthogonality also allows high spectral efficiency.

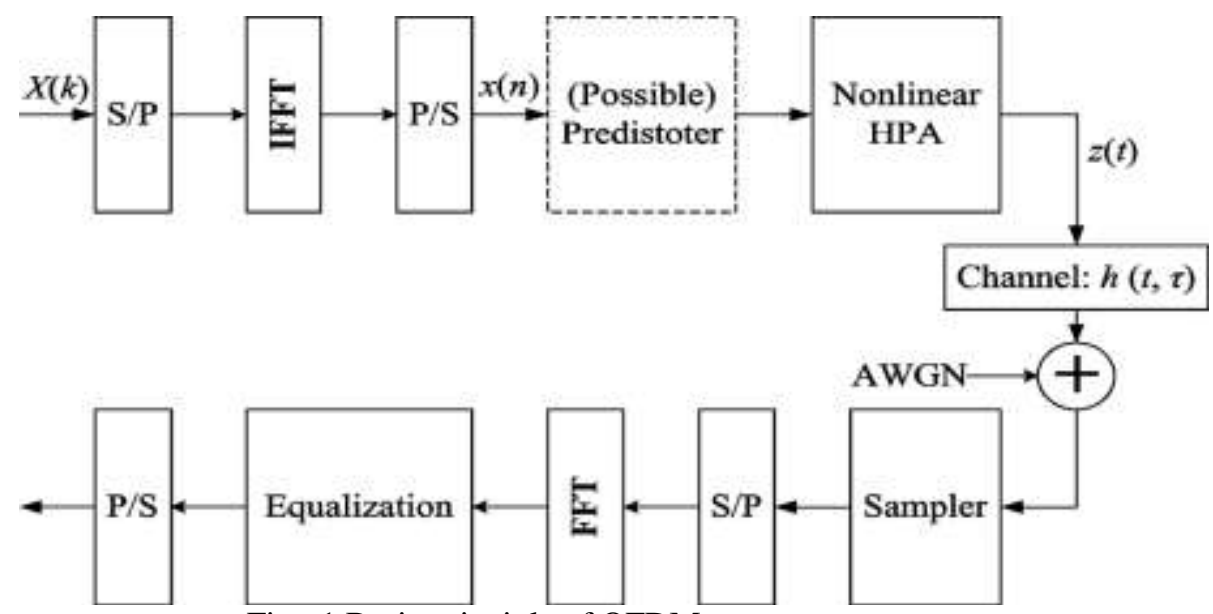

Fig . 1 Basic principle of OFDM system

Orthogonal frequency division multiplexing (OFDM) technique has been accepted for many applications such as mobile and indoor wireless communications.

It is well known that OFDM is spectrally efficient but power inefficient due to the large peak-toaverage power ratio (PAPR) inherent in the OFDM signals, a large PAPR value also makes the signal vulnerable to nonlinearities in the transmission. 
A number of approaches have been proposed for reducing the PAPR of OFDM signals. For example, selective mapping or transformation [1], [2] may statistically reduce the PAPR with a relatively simple implementation cost.

Although these schemes may offer considerable reduction of the PAPR, a rigorous optimization of reduction process becomes computationally challenging for the system with a large number of subcarriers. Systematic coding techniques [3]-[5] may be attractive since they can deterministically bound the PAPR with little computational cost at the transmitter, but designing the low PAPR codes while maintaining a reasonable coding rate becomes quite difficult as the number of subcarriers increases. Probably simplest for the PAPR reduction is digital clipping and filtering of the OFDM signal [6]-[10]. The problem arises, however, that lowpass filtering the clipped OFDM signal samples results in considerable regrowth of peak power in addition to a certain amount of degradation in bit-error performance. In clipping technique [11], OFDM signal peaks larger than some threshold are deliberately clipped off. Even though this is a simple technique, it introduces in-band distortion and out of band noise. In Selective Mapping (SLM) technique the sequence with the lowest PAPR after making the few different phase changes on the identical input data sequence is selected and transmitted. To recover data, the receiver must have knowledge about the generation process of OFDM signal and phase information. This information is known as Side information (SI), which results in some loss of efficiency. To mitigate the performance degradation in the propagation channel, channel coding is usually used in communication systems.

The paper is organized as follows. After the detailed description of the peak to average power ratio in Section 2, the PAPR reduction schemes for OFDM signals is examined in Section 3. Finally conclusions are given in Section 4.

\section{Peak To Average Power Ratio (PAPR)}

An OFDM signal is the sum of $C_{i}$ complex random variables, each of it can be considered as a complex modulated signal at a different frequency. In some cases, all signal components can add up in phase and produce a large output and in some cases, they may cancel each other, producing zero output. Thus, the peak-to-average ratio (PAPR) of an OFDM system is very large. In the transmitter front-end, a power amplifier is required with a wide linear range that can include the peaks in the transmitted waveform. DAC's and ADC's must also have a wide signal range to avoid clipping. The symbols that have a large PAPR are vulnerable to errors.

Peak to Average power Ratio is defined by Muller and Huber (1997) [7]:

$$
P A P R=\frac{\max \left|s_{x}(t)\right|^{2}}{\in\left\{\left|s_{x}(t)\right|^{2}\right\}} t \in[0, T]
$$

where $E$ denotes the expectation and $S_{x}(t)$ is the OFDM transmitted signal. This expectation value is the value that we expect to measure most often if repeated measurements were made on the system. The PAPR also can be expressed in $\mathrm{dB}$ as in equation (2).

$$
P A P R=10 \log \frac{\max \left|s_{x}(t)\right|^{2}}{\left|s_{x}(t)\right|^{2} / N}[d b]
$$

Where $\max \left|S_{x}(t)\right|^{2}$ is the maximum power of the OFDM transmitted signal while $\left|S_{x}(t)\right|^{2} / \mathrm{N}$ is the average power for $\mathrm{N}$ number of carriers.

\subsection{Tone Reservation}

\section{PAPR Reduction Techniques}

In this scheme, some OFDM subcarriers are reserved. These reserved subcarriers don't carry any data information, are only used for reducing PAPR. This method is called Tone Reservation. This technique includes number of set of reservation of tones. By using this technique reserved tones can be used to minimize the PAPR. This method is used for multicarrier transmission and also shows the reserving tones to reduce the PAPR. This technique depends on amount of complexity. When the number of tones is small reduction in PAPR may represent non negligible samples of available bandwidth. Advantage of this tone reservation is very positive that no process is needed at receiver end. And also do not need to transmit the side information along with the transmitted signal. In this technique number of loops are used and the signal will pass from each loop and depending nunber of iterate the output PAPR value will be displayed. 


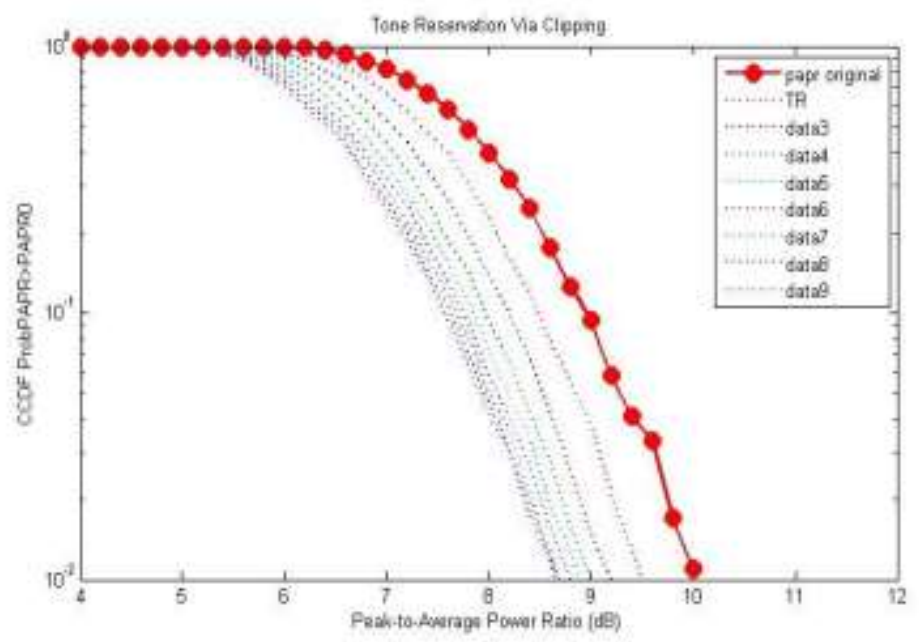

Fig.2 shows PAPR reduction up to $1.5 \mathrm{~dB}$

As per the technique above figure shows the previous simulation by keeping $C R=1$, that why reduction in the PAPR can be done up to $1.5 \mathrm{~dB}$ only but the times always wants some changes so by this technique due to some changes better result can be display. In table the data shows reduction in CCDF of PAPR.

Table-1 Data of Reduction in CCDF for $M=32$

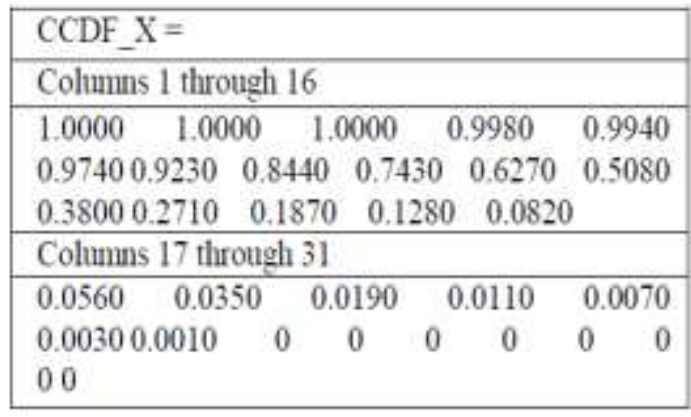

Table-2 Data of Reduction in CCDF for $M=32$

\begin{tabular}{|l|lllll|}
\hline \multicolumn{1}{l}{ CCDF X $=$} \\
\hline \multicolumn{7}{|l|}{ Columns I through 16} \\
\hline 1.0000 & 1.0000 & 1.0000 & 1.0000 & 1.0000 & 1.0000 \\
1.0000 & 1.0000 & 1.0000 & 0.9990 & 0.9970 & 0.9890 \\
0.9720 & 0.9330 & 0.8820 & 0.8280 & & \\
\hline Columns 17 through 31 \\
\hline 0.7470 & 0.6640 & 0.5800 & 0.4880 & 0.3970 & 0.3190 \\
0.2460 & 0.1760 & 0.1260 & 0.0940 & 0.0580 & 0.0410 \\
0.0330 & 0.0170 & 0.0110 & & & \\
\hline
\end{tabular}

The signal generated in BPSK modulation signal then signal passed from 1000 loops. OFDM signal's peaks can be remove at some level by using this technique. In this technique by using previous reservation terms further can be evaluate. Now as per shown the fig. 3 this is the simulation result by using the tone reservation technique to reduce the PAPR. This proposed technique includes signal power and mean power.

Now in this technique OFDM signal is transmitted after performing IFFT.

$X=\operatorname{IFFT}(X * T R) * \sqrt{ } N$

Here as per the signal power and combination of mean power and CR. Reduction in PAPR has been possible. 


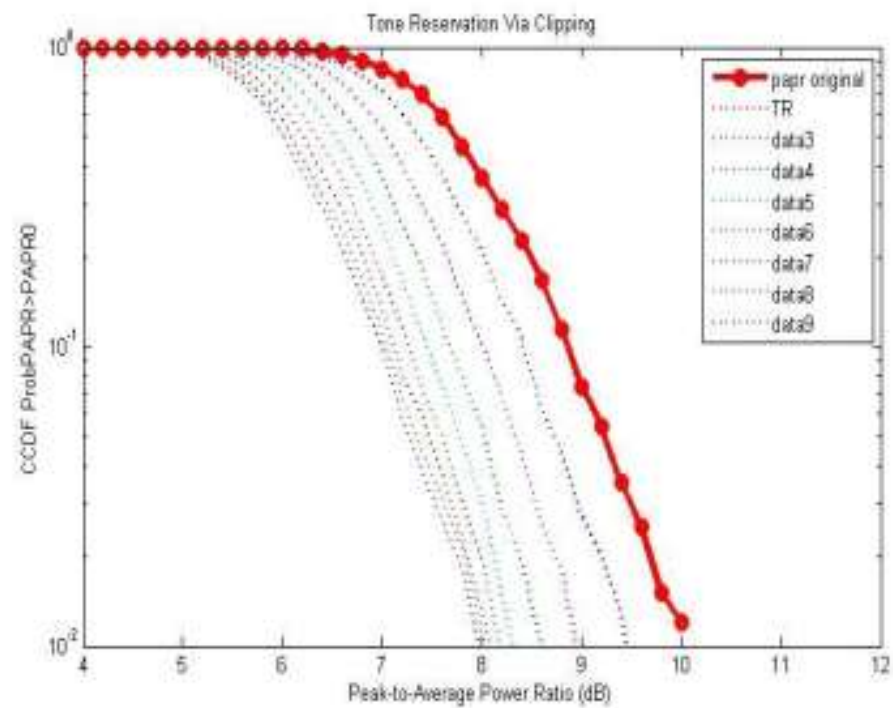

Fig. 3 : Clipping Ratio $(\mathrm{CR})=2$

Here particular in this technique loop is 1000 . And the reducing of data we can see here it is continuous decreasing. The value of CCDF is checked for 32 Times because here $M=32$.

\subsection{Partial Transmit sequence}

For PAPR reduction using partial transmit sequence a typical OFDM system with input data block in $X$ has been partitioned into $M$ disjoint sub-blocks of clusters, which are represented by the vectors $\{X(m), m=0,1, M-1\} \quad[19]$.

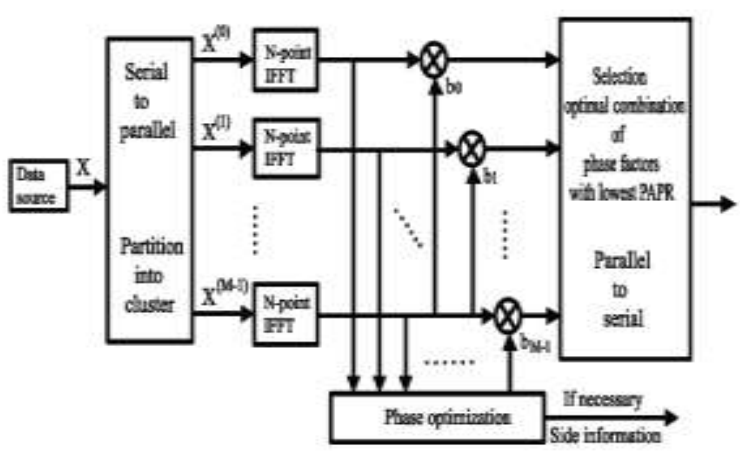

Fig.4: Block Diagram of PTS Technique

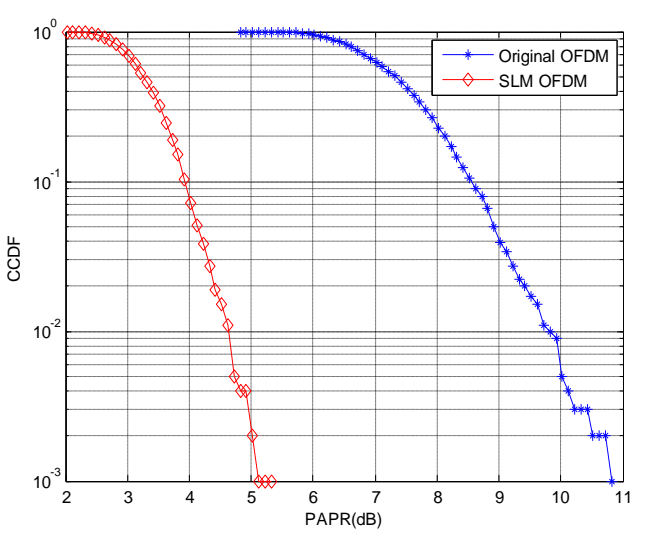

Fig.5: Reduced PAPR using PTS Technique

Therefore

$X=\sum_{M=0}^{M-1} X^{(m)}$

Where

$X(m)=\left[X_{0}(m) X_{1}(m) \quad \ldots \ldots . . X_{\mathrm{N}}-1(m)\right]$ with $X_{\mathrm{k}}(m)=X_{\mathrm{k}}$ or $0(0 \leq m \leq M-1)$. In general, for PTS scheme, the known sub block partioning methods can be classified into three categories: adjacent partition, interleaved partition and pseudo-random partition. Then, the sub blocks $X(m)$ are transformed into $M$ timedomain partial transmit sequences .

$$
x^{(\mathrm{m})}=I F F T_{\mathrm{LNxN}}\left[X^{(\mathrm{m})}\right]
$$

These partial sequences are independently rotated by phase factors

$$
\boldsymbol{b}=\{b m=e j \theta m, m=0,1, \ldots, M-1\} \text {. }
$$


The objective has been to optimally combine the $M$ sub blocks to obtain the time domain OFDM signals with the lowest PAPR.

$$
X=\sum_{M=0}^{M-1} b_{m} X^{(m)}
$$

Assuming that there are $W$ phase angles to be allowed, thus $b m$ has the possibility of $W$ different values. Therefore, there are $W^{\mathrm{m}}$ alternative representations for an OFDM symbol.

The PTS technique significantly reduces the PAPR, but unfortunately, finding the optimal phase factors has been a highly complex problem. In order to reduce the search complexity, the selection of the phase factors has been limited to a set of finite number of elements. The Exhaustive Search Algorithm (ESA) [20] has been employed to find the best phase factor. However, the ESA requires an exhaustive search over all combinations of the allowed phase factors and has exponential search complexity with the number of sub blocks. To reduce the computational complexity, some simplified search techniques have been proposed such as the Iterative Flipping Algorithm (IFA) [21]. Although the IFA significantly reduces the search complexity, there has been some gap between its PAPR reduction performance and that of the ESA. A Cross-Entropy (CE) based method has been proposed by Jung-Cheih Chen [20] for obtaining the optimal phase factors for the PTS technique to reduce the PAPR. Jung-Cheih Chen[22] has proposed a Quantum-Inspired Evolutionary Algorithm(QEA) based method to obtain the optimal phase factor for the PTS technique. Abolfazl [23] has proposed an AutoCorrelation Function (ACF) to develop a new PTS sub blocking technique using Error-Correcting Codes (ECCs). This technique minimizes the number of repeated subcarrier with a sub-block and provides better PAPR reduction than pseudorandom or $\mathrm{m}$-sequence sub blocking.

\subsection{Selective Mapping Technique}

In SLM, the input data sequences have been multiplied by each of the phase sequences to generate alternative input symbol sequences. Each of the alternative input data sequences is made the IFFT operation, and then the one with the lowest PAPR is selected for transmission [11]. A block diagram of the SLM technique has been depicted in Fig.7. Each data block is multiplied by $V$ different phase factors, each of length $N$,

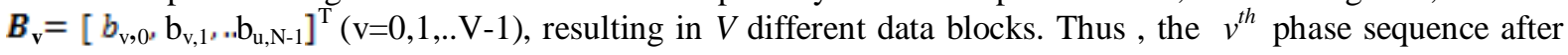

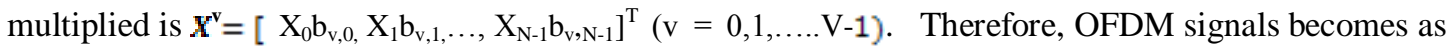
$X^{V}(m)=\frac{1}{\sqrt{N}} \sum_{\mathbb{N}=0}^{N-1} X_{n} b_{n m} e^{j 2 \pi f_{n} t}$

Where $0 \leq t \leq N T, \mathrm{v}=1,2, \ldots \ldots, \mathrm{V}-1$.

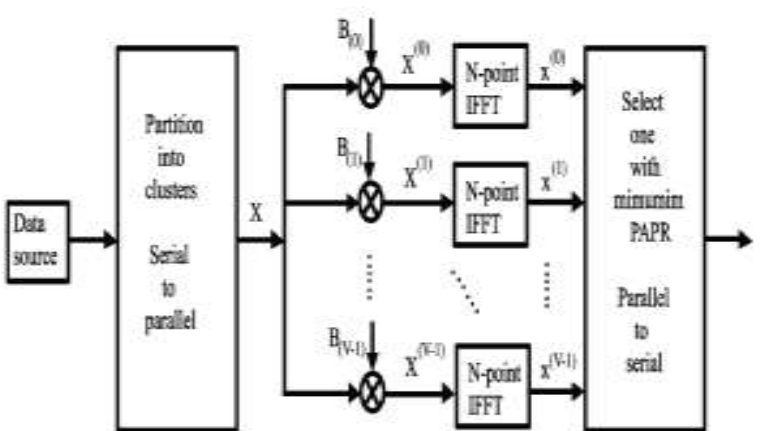

Fig.6. Block Diagram of SLM Technique

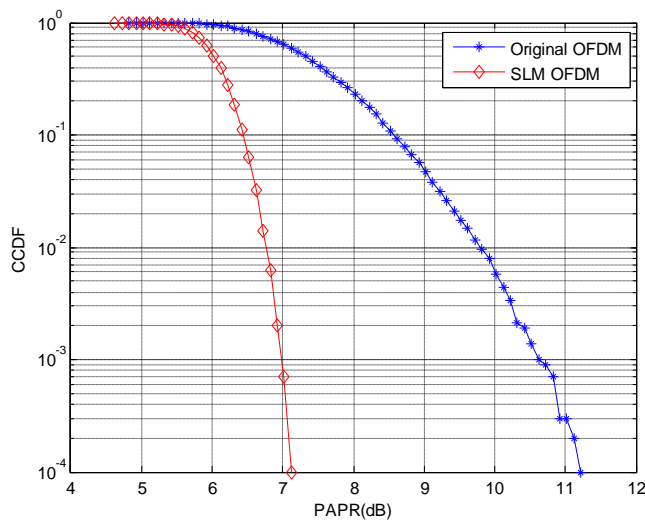

Fig.7 PAPR reduction using SLM

Among the data block $\mathrm{X}^{\mathrm{v}}(\mathrm{v}=0,1, \ldots \mathrm{V}-1)$, only one with the lowest PAPR has been selected for transmission and the corresponding selected phase factors $b_{v, n}$ also should be transmitted to receiver as side information. For implementation of SLM OFDM systems the SLM technique needs $V$ IFFT operation and the number of required bits as side information is $\left[\log _{2} V\right]$ for each data block. Therefore, the ability of PAPR reduction in SLM depends on the number of phase factors $V$ and the design of the phase factors. Some extension of SLM also has been proposed to reduce the computational complexity and number of the bits for side information transmission [16]. Selected Mapping (SLM) has been one of the most popular signal scrambling techniques used to reduce the PAPR of OFDM signals. In symbol scrambling techniques the input data sequence has been "scrambled" using a number of specialized scrambling sequences. The sequence which produces the lowest PAPR is the one used for transmission. The SLM technique proposed by Bauml et al. [14] takes the OFDM subcarrier data block 
to be transmitted and multiples it element-wise by a number of phase adjustment vector sets. The new statistically independent phase adjusted OFDM frames represent the same transmitted information, but have different PAPR values. The OFDM frame that has the lowest PAPR is then selected to be transmitted. As reported in [12] the SLM technique can provide a $0.1 \%$ probability PAPR reduction of $2.8 \mathrm{~dB}$ (from $10.4 \mathrm{~dB}$ to $7.6 \mathrm{~dB}$ ) when applied to 128 -subcarrier QPSK-OFDM symbols. However, one drawback of this technique is that some additional information relating to the phase vector set that produces the lowest PAPR also requires to be transmitted along with the OFDM signal. This extra information increases the overhead.

\section{Conclusions}

In this paper some PAPR reduction techniques for multicarrier transmission have been discussed. Many techniques to reduce the PAPR have been proposed all of which have the potential to provide substantial reduction in PAPR at the cost of loss in data rate, transmit signal power increase, BER increase, computational complexity increase and so on.

\section{REFERENCES}

[1] R. W. Bäuml, R. F. H. Fischer, and J. B. Huber, "Reducing the peak-toaverage power ratio of multicarriermodulation by selected mapping," Electron. Lett., vol. 32, pp. 2056-2057, Oct. 1996.

[2] D. J. G. Mestdagh and P. M. P. Spruyt, “A method to reduce the probability of clipping in DMT-based ransceivers," IEEE Trans. Commun., vol. 44, pp. 1234-1238, Oct. 1996.

[3] R. D. J. van Nee, "OFDM codes for peak-to-average power reduction and error correction," in Proc. IEEE GLOBECOM '96, London, U.K, Nov. 1996, pp. 740-744.

[4] J. A. Davis and J. Jedwab, "Peak-to-mean power control in OFDM,Golay complementary sequences and Reed- Muller codes," IEEE Trans. Inform. Theory, vol. 45, pp. 2397-2417, Nov. 1999.

[5] H. Ochiai and H. Imai, "Block coding scheme based on complementary sequences for multicarrier signals," EICE Trans. Fundamentals, vol. E80-A, pp. 2136-2143, Nov. 1997.

[6] R. O’Neill and L. B. Lopes, "Envelope variations and spectral splatter in clipped multicarrier signals," in Proc. PIMRC '95, vol. 1, Toronto, Canada, Sept. 1995, pp. 71-75.

[7] X. Li and L. J. Cimini Jr, "Effects of clipping and filtering on the performance of OFDM," in Proc. VTC '97, Phoenix, AZ, May 1997, pp. 1634-1638.

[8] D. Wulich and L. Goldfeld, "Reduction of peak factor in orthogonal multicarrier modulation by amplitude limiting and coding," IEEE Trans. Commun., vol. 47, pp. 18-21, January 1999.

[9] D. Kim and G. L. Stüber, "Clipping noise mitigation for OFDM by decision-aided reconstruction,” IEEE Commun. Lett., vol. 3, pp. 4-6, Jan.1999.

[10] R. Dinis and A. Gusmão, "On the performance evaluation of OFDM transmission using clipping techniques," in Proc. IEEE VTC '99 Fall,Amsterdam, The Netherlands, Sept. 1999, pp. 2923-2928.

[11] Xiaodong Li and Leonard J.Cimini(1 998), "Effects of clipping and filtering on the performance of OFDM",IEEE Communication letters,Vol.2, No.5, pp.131-133, May.

[12] T.Ya-fei, D.Rong-hua, Y.Xiao-an and T.Hai-wei,“ PAPR Reduction of OFDM Signals Using Modified Partial Transmit Sequences,” 2nd International Conference on Image and Signal Processing, 2009,CISP’09, pp.1-4 .

[13] J.C.Chen,"Partial Transmit Sequences for Peak-to-Average Power Ratio Reduction of OFDM Signals With the Cross-Entropy Method," IEEE Signal Processing Letters, Vol.16, No.6, June 2009, pp.545-548.

[14] T.Jiang and Y.Wu, "An Overview: Peak-to-Average Power Ratio Reduction Techniques for OFDM Signals," IEEE Transactions on Broadcasting, Vol.54, No.2, June 2008, pp.257-268.

[15] S.H.Han and J.H.Lee, "PAPR Reduction of OFDM Signals using a Reduced Complexity PTS Technique," IEEE Signal Processing Letters, Vol.11, No.11, Nov.2004, pp.887-890.

[16] R.W.Bauml, R.F.H.Fisher and J.B.Huber, "Reducing the Peak-to- Average Power Ratio of Multicarrier Modulation by Selected Mapping," IEEE Electronic Letters, Vol.32, Oct. 1996, pp.2056-2057. 